\title{
Critical exponent for the Cauchy problem to the weakly coupled damped wave system
}

\author{
Kenji Nishihara ${ }^{1}$ \\ Faculty of Political Science and Economics, Waseda University \\ and \\ Yuta Wakasugi \\ Department of Mathematics, Graduate School of Science, Osaka University
}

\section{Introduction}

In this note we consider the Cauchy problem for a weakly coupled damped wave system

$$
\left\{\begin{array}{c}
u_{t t}-\Delta u+u_{t}=|v|^{p}, \\
v_{t t}-\Delta v+v_{t}=|u|^{q},
\end{array} \quad(t, x) \in \mathbf{R}_{+} \times \mathbf{R}^{N}\right.
$$

and the data

$$
\left(u, u_{t}, v, v_{t}\right)(0, x)=\varepsilon\left(u_{0}, u_{1}, v_{0}, v_{1}\right)(x), \quad x \in \mathbf{R}^{N},
$$

with $\left(u_{0}, u_{1}, v_{0}, v_{1}\right) \in\left[H^{1} \times L^{2}\right]^{2}:=\left(H^{1} \times L^{2}\right) \times\left(H^{1} \times L^{2}\right)$ and small constant $\varepsilon$. Our main interest is to obtain the critical exponents $(p, q)$ in the sense that, in the supercritical exponents there exists a (unique) solution $(u, v)$ of (1.1)-(1.2), and in the critical or subcritical exponents the local-in-time solution blows up within a finite time for suitable data. In the latter case we also have an interest in the estimate of lifespan $T_{\varepsilon}$.

For the single damped wave equation

$$
U_{t t}-\Delta U+U_{t}=|U|^{\rho}, \quad(t, x) \in \mathbf{R}_{+} \times \mathbf{R}^{N},
$$

the critical exponent is the Fujita exponent

$$
\rho_{F}(N)=1+\frac{2}{N}
$$

as same as for the corresponding parabolic equation, named after his pioneering work [2] (for (1.3) see Todorova and Yordanov [19], Zhang [21], Hayashi, Kaikina and Naumkin [3], Nishihara [10] etc.). This is easily imagined by the diffusion phenomena of solutions of damped wave equation. This property is well-understood by the solution formula of the Cauchy problem for the linear damped wave equation

$$
\left\{\begin{array}{l}
V_{t t}-\Delta V+V_{t}=0, \quad(t, x) \in \mathbf{R}_{+} \times \mathbf{R}^{N} \\
\left(V, V_{t}\right)(0, x)=(0, g)(x), \quad x \in \mathbf{R}^{N}
\end{array}\right.
$$

\footnotetext{
${ }^{1}$ This work was supported in part by Grant-in-Aid for Scientific Research (C) 25400184 of Japan Society for the Promotion of Science.
} 
In fact, the solution denoted by $\left[S_{N}(t) g\right](x)$ is given, e.g. when $N=3$, by

$$
\left[S_{3}(t) g\right](x)=\frac{e^{-t / 2}}{4 \pi t} \partial_{t} \int_{|z| \leq t} I_{0}\left(\frac{1}{2} \sqrt{t^{2}-|z|^{2}}\right) g(x+z) d z
$$

where $I_{\nu}(y)$ is the modified Bessel function of order $\nu$

$$
I_{\nu}(y)=\sum_{m=0}^{\infty} \frac{1}{m !(m+\nu) !}\left(\frac{y}{2}\right)^{2 m+\nu} .
$$

In (1.6) differentiating in $t$ and using $I_{0}(0)=1, I_{0}^{\prime}(y)=I_{1}(y)$, we have

$$
\begin{aligned}
& {\left[S_{3}(t) g\right](x)} \\
& \quad=e^{-t / 2} \cdot \frac{t}{4 \pi} \int_{S^{2}} g(x+t \omega) d \omega+\frac{e^{-t / 2}}{8 \pi} \int_{|z| \leq t} I_{1}\left(\frac{1}{2} \sqrt{t^{2}-|z|^{2}}\right) \frac{g(x+z) d z}{\sqrt{t^{2}-|z|^{2}}} \\
& \quad=: e^{-t / 2} \cdot\left[W_{3}(t) g\right](x)+\left[J_{30}(t) g\right](x) .
\end{aligned}
$$

Note that $W_{3}(t) g$ is the Kirchhoff formula for the wave equation without damping. Moreover, by the expansion formula

$$
I_{\nu}(y)=\frac{e^{y}}{\sqrt{2 \pi y}}\left(1+O\left(\frac{1}{y}\right)\right), \quad y \rightarrow \infty
$$

the remainder term $J_{30}(t) g$ is estimated for $1 \leq q \leq p \leq \infty$ as

$$
\begin{aligned}
\left\|J_{30}(t) g\right\|_{L^{p}} & \leq C(1+t)^{-\frac{3}{2}\left(\frac{1}{q}-\frac{1}{p}\right)}\|g\|_{L^{q}}, \quad t \geq 0, \\
\left\|\left(J_{30}(t)-e^{t \Delta}\right) g\right\|_{L^{p}} & \leq C t^{-\frac{3}{2}\left(\frac{1}{q}-\frac{1}{p}\right)-1}\|g\|_{L^{q}}, \quad t>0,
\end{aligned}
$$

for some positive constant $C$, where

$$
\left[e^{t \Delta} g\right](x)=\int_{\mathbf{R}^{3}} G(t, x-y) g(y) d y=\int_{\mathbf{R}^{3}}(4 \pi t)^{-\frac{3}{2}} e^{-\frac{|x-y|^{2}}{4 t}} g(y) d y .
$$

Thus, the solution of damped wave equation is generally decomposed to the wave part decaying fast and the diffusive part. Therefore, as time tends to infinity, the solution of damped wave equation behaves like that of corresponding diffusive (parabolic) equation. This phenomena is called the diffusion phenomena. In other words, the solution of damped wave equation has the properties of wave equation qualitatively, and behaves like the diffusion quantitatively (see Nishihara [10], Sakata and Wakasugi [16]).

We now go back to the system. By the diffusion phenomena stated above, we can expect that the problem (1.1)-(1.2) has the same critical exponents as the corresponding parabolic system

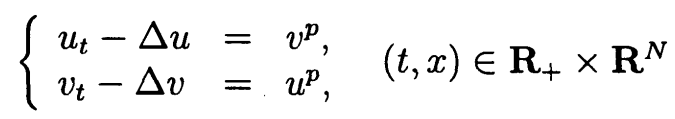


with $(u, v)(0, x)=\left(u_{0}, v_{0}\right)(x) \geq 0, \quad x \in \mathbf{R}^{N}$. Note that, since the data are nonnegative, $|v|^{p}=v^{p}$ etc. and $|\cdot|$ are removed. For the parabolic system (1.10) with $p, q>0, p q>1$, Escobedo and Herrero [1] showed that the critical exponents are given by

$$
\alpha=\frac{N}{2}, \quad \alpha:=\max \left(\alpha_{1}, \alpha_{2}\right):=\max \left(\frac{p+1}{p q-1}, \frac{q+1}{p q-1}\right) .
$$

Our aim is to show that (1.11) still give the critical exponents for (1.1)-(1.2).

First we show how to get (1.11), referring the general system

$$
\left\{\begin{array}{l}
u_{t t}-\Delta u+u_{t}=|u|^{p_{11}}|v|^{p_{12}} \\
v_{t t}-\Delta v+v_{t}=|u|^{p_{21}}|v|^{p_{22}}
\end{array}\right.
$$

following Ogawa and Takeda [14]. If both $u$ and $v$ behave like the Gauss kernel $G$ in the supercritical exponents, then for $i=1,2$

$$
\int_{0}^{\infty}\left\||u|^{p_{i 1}}|v|^{p_{i 2}}(t, \cdot)\right\|_{L^{1}} d t \leq C \int_{0}^{\infty}(1+t)^{-\frac{N}{2}\left(p_{i 1}+p_{i 2}-1\right)} d t<\infty
$$

which yields $\frac{N}{2}\left(p_{11}-1+p_{12}\right)>1, \frac{N}{2}\left(p_{21}+p_{22}-1\right)>1$ or

$$
\frac{N}{2}(P-E)\left(\begin{array}{l}
1 \\
1
\end{array}\right)>\left(\begin{array}{l}
1 \\
1
\end{array}\right), \quad P=\left(\begin{array}{ll}
p_{11} & p_{12} \\
p_{21} & p_{22}
\end{array}\right) .
$$

Formally, multiply $(P-E)^{-1}$, then

$$
\frac{N}{2}\left(\begin{array}{c}
1 \\
1
\end{array}\right)>(P-E)^{-1}\left(\begin{array}{c}
1 \\
1
\end{array}\right)=:\left(\begin{array}{c}
\alpha_{1} \\
\alpha_{2}
\end{array}\right)
$$

and obtain the supercritical exponents $\alpha=\max \left(\alpha_{1}, \alpha_{2}\right)<\frac{N}{2}$. Here, note that (1.12) is not necessarily equivalent to (1.13). But, by these we formally get (1.11). In fact, for (1.1)

$$
P=\left(\begin{array}{ll}
0 & p \\
q & 0
\end{array}\right), \text { and }\left(\begin{array}{c}
\alpha_{1} \\
\alpha_{2}
\end{array}\right)=(P-E)^{-1}\left(\begin{array}{l}
1 \\
1
\end{array}\right)=\frac{1}{p q-1}\left(\begin{array}{c}
p+1 \\
q+1
\end{array}\right) .
$$

We now concentrate to (1.1)-(1.2) with $q \geq p$ without loss of generality. So, the critical exponents are expected to

$$
\alpha=\frac{q+1}{p q-1}=\frac{N}{2} \text { or } q\left(p-\frac{2}{N}\right)=1+\frac{2}{N}\left(=\rho_{F}(N)\right) .
$$

When $N=1,2,3$, we can reformulate (1.1)-(1.2) to the system of integral equations

$$
\left\{\begin{array}{l}
u(t, \cdot)=S_{N}(t)\left(u_{0}+u_{1}\right)+\partial_{t}\left(S_{N}(t) u_{0}\right)+\int_{0}^{t} S_{N}(t-s)|v|^{p}(s, \cdot) d s \\
v(t, \cdot)=S_{N}(t)\left(v_{0}+v_{1}\right)+\partial_{t}\left(S_{N}(t) v_{0}\right)+\int_{0}^{t} S_{N}(t-s)|u|^{q}(s, \cdot) d s .
\end{array}\right.
$$


Since $S_{N}(t) g$ includes the wave part, when $N \geq 4$, the loss of regularity causes difficulty and (1.15) is not available. However, in low dimension it works usefully. Roughly speaking, the followings are known.

(i) When $\alpha<\frac{N}{2}$ (supercritical exponents), if $0<\varepsilon \ll 1$, then there exists a unique global-in-time solution exists and its asymptotic profiles are obtained. See Sun and Wang [17], Nishihara [12], Takeda [18] (cf. Narazaki [9], Ogawa and Takeda [14, 15]).

On the other hand,

(ii) When $\alpha \leq \frac{N}{2}$ (critical and subcritical exponents), without restriction on the space dimension, the solution does not exists globally for suitable data. See Sun and Wang [17].

Thus, we now reach to state our problem and results. One is to obtain the global-intime solution in any space dimension, and the other is to obtain the estimate of lifespan $T_{\varepsilon}$ in the subcritical exponents.

Theorem 1.1 ([13]) Suppose that $\left(u_{0}, u_{1}, v_{0}, v_{1}\right) \in\left[H^{1} \times L^{2}\right]^{2}:=\left[H^{1} \times L^{2}\right] \times\left[H^{1} \times L^{2}\right]$ and $1<p, q<\frac{N}{[N-2]_{+}}\left(=\infty\right.$ if $N=1,2$ and $=\frac{N}{N-2}$ if $N \geq 3$.)

(i) (Supercritical) Let $\alpha<N / 2$ and the data be compactly supported with $0<\varepsilon \ll 1$. Then there exists a unique weak solution $(u, v) \in\left[C\left([0, \infty) ; H^{1}\right) \cap C^{1}\left([0, \infty) ; L^{2}\right)\right]^{2}$ of (1.1)-(1.2) with compact support.

(ii) (Subcritical) Let $\alpha>N / 2$ and $\int_{\mathbf{R}^{N}}\left(u_{0}+u_{1}\right)(x) d x>0, \quad \int_{\mathbf{R}^{N}}\left(v_{0}+v_{1}\right)(x) d x>0$. Then the solution does not exist globally in time and the blow-up time $T_{\varepsilon}$ is estimated as

$$
T_{\varepsilon} \leq C \varepsilon^{-1 / \kappa}, \kappa=\alpha-\frac{N}{2}(C>0: \text { constant }) .
$$

Remark. (i) For the existence of global-in-time solution we have assumed the compactness on the data for simplicity. For more wider class of data we can obtain the global existence.

(ii) We believe the estimate (1.16) is optimal, but the estimate from below has a gap to (1.16). In the critical exponent case the estimate is not obtained.

In the supercritical exponents we apply the weighted energy method, originally developed by Todorova and Yordanov [19]. In the subcritical exponents the test function method is applied, which is developed by Mitidieri and Pohozaev [8] for several evolution equations, Zhang [21] for the damped wave equation, and Ikeda and Wakasugi [5] for the estimate of lifespan. See also Li and Zhou [7].

\section{Existence of global-in-time solution}

Consider (1.1)-(1.2) in case of the supercritical exponents $(p, q)$ which satisfy

$$
q\left(p-\frac{2}{N}\right)>1+\frac{2}{N}=\rho_{F}(N)
$$


by (1.14). The region of $(p, q)$ in (2.1) is decomposed to

(I) $p, q>\rho_{F}(N)$ and (II) $\max \left(1, \frac{2}{N}\right)<p \leq \rho_{F}(N)<q$.

In Case (I) we can easily expect that the solution $(u, v)$ behaves as the Gauss kernel and

$$
\|(u, v)(t)\|_{L^{r}}=O\left(t^{-\frac{N}{2}\left(1-\frac{1}{r}\right)}\right) \quad \text { as } t \rightarrow \infty
$$

if the data are in $L^{1} \cap L^{\infty}$.

However, in Case (II), because of $p \leq \rho_{F}(N)$, (2.2) will not hold. To apply the weighted energy method we need the expected decay rate of solutions in $L^{2}$. So, let us discuss the estimates of $\|u(t)\|_{L^{2}},\|v(t)\|_{L^{2}}$, heuristically, when $\frac{2}{N}<p<\rho_{F}(N)<q$. Since $q>\rho_{F}(N)$, assume for a moment

$$
\int_{0}^{\infty}\left\||u|^{q}(t)\right\|_{L^{1}} d t<\infty
$$

Then, by $(1.1)_{2}$ (second equation in (1.1))

$$
\|v(t)\|_{L^{r}}=O\left(t^{-\frac{N}{2}\left(1-\frac{1}{r}\right)}\right), \quad t \rightarrow \infty(1 \leq r \leq \infty)
$$

if $v_{0}, v_{1}$ is in $L^{1} \cap L^{\infty}$. Therefore, by $(1.1)_{1}$ and diffusion phenomena,

$$
\begin{aligned}
\|u(t)\|_{L^{1}} & \leq C\left\|u_{0}, u_{1}\right\|_{L^{1}}+C \int_{0}^{t}\left\|G(t-s) *|v|^{p}(s)\right\|_{L^{1}} d s \\
& \leq C\left\|u_{0}, u_{1}\right\|_{L^{1}}+C \int_{0}^{t}\|v(s)\|_{L^{\infty}}^{p-1}\|v(s)\|_{L^{1}} d s \\
& \leq C\left\|u_{0}, u_{1}\right\|_{L^{1}}+C \int_{0}^{t}(1+s)^{-\frac{N}{2}(p-1)} d x=O\left(t^{\frac{N}{2}\left(1+\frac{2}{N}-p\right)}\right),
\end{aligned}
$$

which may grow up as $t \rightarrow \infty$. While,

$$
\begin{aligned}
\|u(t)\|_{L^{\infty}} \leq & C(1+t)^{-\frac{N}{2}}\left\|u_{0}, u_{1}\right\|_{L^{1} \cap L^{\infty}} \\
& +C \int_{0}^{\frac{t}{2}}(1+t-s)^{-\frac{N}{2}}\left\||v|^{p}(s)\right\|_{L^{1}} d s+C \int_{\frac{t}{2}}^{t}\left\|\left.v\right|^{p}(s)\right\|_{L^{\infty}} d s \\
\leq & C(1+t)^{-\frac{N}{2}}\left\|u_{0}, u_{1}\right\|_{L^{1} \cap L^{\infty}} \\
& +C(1+t)^{-\frac{N}{2}} \int_{0}^{\frac{t}{2}}(1+s)^{-\frac{N}{2}(p-1)} d s+C \int_{\frac{t}{2}}^{t}(1+s)^{-\frac{N}{2} p} d s \\
= & O\left(t^{-\frac{N}{2}\left(p-\frac{2}{N}\right)}\right)
\end{aligned}
$$

which decays as $t \rightarrow \infty$. Note that both (2.5) and (2.6) recover (2.3) by $(1.1)_{2}$. Hence, by interpolation we can expect

$$
\|u(t)\|_{L^{2}} \leq\|u(t)\|_{L^{\infty}}^{\frac{1}{2}}\|u(t)\|_{L^{1}}^{\frac{1}{2}}=O\left(t^{-\frac{N}{2}\left(p-\frac{2}{N}-\frac{1}{2}\right)}\right)=: O\left(t^{-\frac{k}{2}}\right) .
$$

The proof of local existence of solution is standard and omitted. Let us go to the a priori estimate on the solution $(u, v) \in\left[C\left([0, T) ; H^{1}\right) \cap C^{1}\left([0, T) ; L^{2}\right)\right]^{2}$ with compact support. Our expectation (2.7) yields the setting

$$
\begin{aligned}
& W_{u}(s)=(1+s)^{k+1-(p+1) \delta} \int_{\mathbf{R}^{N}} e^{2 \psi}\left(u_{t}^{2}+|\nabla u|^{2}\right)(s, x) d x+(1+s)^{k-(p+1) \delta} \int_{\mathbf{R}^{N}} e^{2 \psi} u^{2}(s, x) d x \\
& W_{v}(s)=(1+s)^{\frac{N}{2}+1-\delta} \int_{\mathbf{R}^{N}} e^{2 \psi}\left(v_{t}^{2}+|\nabla v|^{2}\right)(s, x) d x+(1+s)^{\frac{N}{2}-\delta} \int_{\mathbf{R}^{N}} v^{2}(s, x) d x
\end{aligned}
$$


and

$$
M(t)=\sup _{0 \leq s \leq t}\left(W_{u}(s)+W_{v}(s)\right)
$$

when $(p, q)$ is in Case (II). Here, in the weight $e^{2 \psi}, \psi$ has the form

$$
\psi=\psi(t, x)=\frac{|x|^{2}}{4(2+\lambda)(1+t)}, \quad \text { with } \quad \lambda=\frac{8 \delta}{3 N-4 \delta}
$$

and $\delta>0$ is an arbitrarily small constant. This kind of weight has been developed by $[19,4,6,11,20]$ etc.

Then, by standard but rather complicated calculations, we can derive

$$
M(t) \leq C \varepsilon^{2} I_{0}+C\left(M(t)^{p}+M(t)^{\frac{p+1}{2}}+M(t)^{q}+M(t)^{\frac{q+1}{2}}\right),
$$

which shows the desired estimate if $0<\varepsilon \ll 1$, where

$$
I_{0}=\int_{\mathbf{R}^{N}} e^{2 \psi(0, x)}\left(u_{0}^{2}+\left|\nabla u_{0}\right|^{2}+u_{1}^{2}+v_{0}^{2}+\left|\nabla v_{0}\right|^{2}+v_{1}^{2}\right)(x) d x<\infty .
$$

Thus we can get Theorem $1.1(i)$. The details are referred in [13].

\section{Estimate on the lifespan}

In this section we consider the case of subcritical exponents. First, introduce the test function

$$
\psi_{\tau, R}(t, x):=\eta_{\tau}(t) \phi_{R}(x):=\eta\left(\frac{t}{\tau}\right) \phi\left(\frac{x}{R}\right)
$$

where

$$
\eta(t)= \begin{cases}1 & (0 \leq t \leq 1 / 2), \\ \frac{\exp \left(-1 /\left(1-t^{2}\right)\right)}{\exp \left(-1 /\left(t^{2}-1 / 4\right)\right)+\exp \left(-1 /\left(1-t^{2}\right)\right)} & (1 / 2<t<1), \\ 0 & (t \geq 1)\end{cases}
$$

and $\phi(x)=\eta(|x|)$. Since we already know no global-in-time solution, $(u, v)$ is a weak solution of $(1.1)$ in $\left[0, T_{\varepsilon}\right)$ and $T_{\varepsilon}$ is the lifespan defined by

$$
T_{\varepsilon}=\sup _{0<T<\infty}\left\{T ; \text { there exists a weak solution }(u, v) \in\left[C\left([0, T) ; H^{1}\right) \cap C^{1}\left([0, T) ; L^{2}\right)\right]^{2}\right\}
$$

and let $\tau \in\left(\tau_{0}, T_{\varepsilon}\right), R \geq R_{0}$ for large parameters $\tau_{0}, R_{0}$. Then, define

$$
V_{\tau, R}=\int_{0}^{\tau} \int_{\mathbf{R}^{N}}|v|^{p} \psi_{\tau, R}(t, x) d x d t, \quad U_{\tau, R}=\int_{0}^{\tau} \int_{\mathbf{R}^{N}}|u|^{q} \psi_{\tau, R}(t, x) d x d t
$$

and

$$
S_{R}=\int_{\mathbf{R}^{N}}\left(v_{0}+v_{1}\right)(x) \phi_{R}(x) d x, \quad T_{R}=\int_{\mathbf{R}^{N}}\left(u_{0}+u_{1}\right)(x) \phi_{R}(x) d x .
$$


Since $(u, v)$ is a weak solution, by integral by parts in $(1.1)_{1}$

$$
U_{\tau, R}+\varepsilon S_{R}=\int_{0}^{\tau} \int_{\mathbf{R}^{N}} v\left(\partial_{t}^{2}-\Delta-\partial_{t}\right) \psi_{\tau, R} d x d t=: K_{1}+K_{2}+K_{3}
$$

For an example,

$$
\begin{aligned}
K_{3} & \leq C \tau^{-1} \int_{0}^{\tau} \int_{\mathbf{R}^{N}}\left|v \| \eta^{\prime}(t / \tau)\right| \phi_{R}(x) d x d t \leq C \tau^{-1} V_{\tau, R}^{1 / p}\left(\int_{0}^{\tau} \int_{|x| \leq R} d x d t\right)^{1 / p^{\prime}} \\
& \leq C \tau^{-1+\frac{1}{p^{\prime}}} R^{\frac{N}{p^{\prime}}} V_{\tau, R}^{1 / p}, \quad\left(\frac{1}{p}+\frac{1}{p^{\prime}}=1\right),
\end{aligned}
$$

where we used $\left|\eta^{\prime}(t)\right| \leq C|\eta(t)|^{1 / p}$. Both $K_{1}$ and $K_{2}$ have similar estimates. Putting $\tau_{0}=R_{0}^{2}, \tau=R^{2}$, we have

$$
U_{\tau, R}+\varepsilon S_{R} \leq C \tau^{-1+\frac{1}{p^{\prime}}+\frac{N}{2 p^{\prime}}} \cdot V_{\tau, R}^{1 / p}
$$

Similarly,

$$
V_{\tau, R}+\varepsilon T_{R} \leq C \tau^{-1+\frac{1}{q^{\prime}}+\frac{N}{2 q^{\prime}}} \cdot V_{\tau, R}^{1 / q}\left(\frac{1}{q}+\frac{1}{q^{\prime}}=1\right) .
$$

Substituting $V_{\tau, R}$ into (3.1) we have

$$
\varepsilon S_{R} \leq C \tau^{-1+\frac{1}{p^{\prime}}+\frac{N}{2 p^{\prime}}+\left(-1+\frac{1}{q^{\prime}}+\frac{N}{2 q^{\prime}}\right) \frac{1}{p}} \cdot U_{\tau, R}^{\frac{1}{p q}}-U_{\tau, R}
$$

Here, since $S_{R} \geq c_{0}>0\left(R \geq R_{0}\right)$, we apply the elementary inequality in $y$

$$
A y^{b}-y \leq A^{\frac{1}{1-b}}(0<b<1, A>0: \text { constants, } y \geq 0)
$$

to (3.3) and get, after simple calculations of the exponent of $\tau$,

$$
\varepsilon \leq C \tau^{-\kappa}, \kappa=\alpha-\frac{N}{2} .
$$

Finally, letting $\tau \rightarrow T_{\varepsilon}-0$ we conclude (1.16) in Theorem 1.1 (ii).

\section{References}

[1] M. Escobedo and M. A. Herrero, Boundedness and blow up for a semilinear reactiondiffusion system, J. Differential Equations 89 (1991), 176-202.

[2] H. Fujita, On the blowing up of solutions of the Cauchy problem for $u_{t}=\Delta u+u^{1+\alpha}$, J. Fac. Sci. Univ. Tokyo, Sec. 1. 13 (1966), 109-124.

[3] N. Hayashi, E. I. Kaikina and P. I. Naumkin, Damped wave equation with a critical nonlinearity, Trans. Amer. Math. Soc. 358 (2006), 1165 - 1185. 
[4] R. Ikehata, G. Todorova and B. Yordanov, Critical exponent for semilinear wave equations with space-dependent potential, Funk. Ekvac. 52 (2009), 411-435.

[5] M. Ikeda and Y. Wakasugi, A note on the lifespan of solutions to the semilinear damped wave equation, Proc. Amer. Math. Soc. (in press).

[6] J. Lin, K. Nishihara and J. Zhai, $L^{2}$ estimates of solutions for the damped wave equations with space-time dependent damping term, J. Differential Equations 248 (2010), 403-422.

[7] T.-T. Li and Y. Zhou, Breakdown of solutions to $\square u+u_{t}=|u|^{1+\alpha}$, Discrete Cont. Dynam. Syst. 1 (1995), 503-520.

[8] E. Mitidieri and S. I. Pohozaev, A priori estimates and the absence of solutions of nonlinear partial differential equations and inequalities, Tr. Mat. Inst Steklova 234 (2001), 1 - 384. Translation in Proc. Steklov Inst. Math. 234 ((2001), 1 - 362.

[9] T. Narazaki, $L^{p}-L^{q}$ estimates for damped wave equations and their applications to semi-linear problem, J. Math. Soc. Japan 56 (2004), 585-626.

[10] K. Nishihara, $L^{p}-L^{q}$ estimates of solutions to the damped wave equation in 3dimensional space and their application, Math. Z. 244 (2003), 631-649.

[11] K. Nishihara, Decay properties for the damped wave equation with space dependent potential and absorbed semilinear term, Commun. Partial Differential Equations 35 (2010), 1402-1418.

[12] K. Nishihara, Asymptotic behavior of solutions for a system of semilinear heat equations and the corresponding damped wave system Osaka J. Math. 49 (2012), 331 348.

[13] K. Nishihara and Y. Wakasugi, Critical exponent for the Cauchy problem to the weakly coupled damped wave system, Nonlinear Analysis 108 (2014), 249 - 259.

[14] T. Ogawa and H. Takeda, Global existence of solutions for a system of nonlinear damped wave equations, Differential Integral Equations 23(2010), 635-657.

[15] T. Ogawa and H. Takeda, Large time behavior of solutions for a system of nonlinear damped wave equations, J. Differential Equations 251 (2011), 3090 - 3113.

[16] S. Sakata and Y. Wakasugi, Movement of time-delayed hot spots in Euclidean space, preprint.

[17] F. Sun and M. Wang, Existence and nonexistence of global solutions for a nonlinear hyperbolic system with damping, Nonlinear Anal. 66 (2007), 2889 - 2910.

[18] H. Takeda, Global existence and nonexistence of solutions for a system of nonlinear damped wave equations, J. Math. Anal. Appl. 360 (2009), 631-650. 
[19] G. Todorova and B. Yordanov, Critical exponent for a nonlinear wave equation with damping, J. Differential Equations 174 (2001), 464-489.

[20] Y. Wakasugi, Small data global existence for the semilinear wave equation with space-time dependent damping, J. Math. Anal. Appl. 393 (2012), 66 - 79.

[21] Q. Zhang, A blow-up result for a nonlinear wave equation with damping: The critical case, C. R. Acad. Sci. Paris 333 (2001), 109-114. 